\title{
Synergistic effect of iontophoresis and chemical enhancers on transdermal permeation of tolterodine tartrate for the treatment of overactive bladder
}

\author{
D. Prasanthi, P. K. Lakshmi \\ G. Pulla Reddy College of Pharmacy, Osmania University, Hyderabad, India
}

\section{ABSTRACT}

Purpose: The objective of the study was to evaluate the synergistic transdermal permeation effect of chemical enhancers and iontophoresis technique on tolterodine tartrate (TT) transdermal gel and to evaluate its pharmacokinetic properties.

Materials and Methods: Taguchi robust design was used for optimization of formulations. Skin permeation rates were evaluated using the Keshary-chein type diffusion cells in order to optimize the gel formulation. In-vivo studies of the optimized formulation were performed in a rabbit model and histopathology studies of optimized formulation were performed on rats.

Results: Transdermal gels were formulated successfully using Taguchi robust design method. The type of penetration enhancer, concentration of penetration enhancer, current density and pulse on/off ratio were chosen as independent variables. Type of penetration enhancer was found to be the significant factor for all the responses. Permeation parameters were evaluated when maximum cumulative amount permeated in 24 hours $\left(Q_{24}\right)$ was $145.71 \pm 2.00 \mu \mathrm{g} / \mathrm{cm}^{2}$ by CIT4 formulation over control $\left(91.89 \pm 2.30 \mu \mathrm{g} / \mathrm{cm}^{2}\right)$. Permeation was enhanced by 1.75 fold by CIT4 formulation. Formulation CIT4 containing nerolidol $(5 \%)$ and iontophoretic variables applied $\left(0.5 \mathrm{~mA} / \mathrm{cm}^{2}\right.$ and pulse on/off ratio 3:1) was optimized. In vivo studies with optimized formulation CIT4 showed increase in AUC and T1/2 when compared to oral suspension in rabbits. The histological studies showed changes in dermis indicating the effect of penetration enhancers and as iontophoresis was continued only for two cycles in periodic fashion so it did not cause any skin damage observed in the slides.

Conclusion: Results indicated that iontophoresis in combination with chemical enhancers is an effective method for transdermal administration of TT in the treatment of overactive bladder.

\section{ARTICLE INFO}

\section{Key words:}

Iontophoresis; Administration, Cutaneous; tolterodine [Sup-

plementary Concept];

Urinary Bladder, Overactive

Int Braz J Urol. 2013; 39: 63-76

Submitted for publication: January 23, 2012

Accepted after revision:

September 24, 2012

\section{INTRODUCTION}

Overactive bladder disorder is a condition characterized by involuntary detrusor contractions that may occur spontaneously or may be provoked (by rapid filling, alterations of posture, coughing, walking and jumping). An overactive bladder of neurogenic origin is referred to as hyperflexic disorder and one that is nonneurogenic is referred to as an unstable disorder (1). Its symptoms include frequent urination, an urge to urinate immediately and urinary incontinence. It often requires long term treatment to maintain control of symptoms (2). Tolterodine tartrate (TT), a synthetic tertiary 
amine antimuscarinic agent is a genitourinary antispasmodic. It decreases contraction of the detrusor muscle of normal and overactive urinary bladder. It has been shown to exhibit functional selectivity for urinary bladder over secretory (e.g., salivary) glands. It is used for the management of symptoms associated with both neurogenic and non-neurogenic overactive bladder (3).

TT is available as conventional tablets and extended release capsules. However, its oral administration is limited on account of its dose-related adverse side effects, including dry mouth, tachycardia, dizziness and gastrointestinal obstructive disorder. TT is extensively metabolized following oral administration, and its major metabolite acts similarly as the parent substance on receptors, which restricts its application in patients with liver cirrhosis (4). This originated the need for an alternative route of administration, which can bypass the hepatic first-pass metabolism. Transdermal route could be an alternative route for these patients, because it bypasses first-pass metabolism, minimizes the gastrointestinal side effects, increases patient compliance, maintains a constant drug level in plasma and makes it possible to interrupt or terminate treatment when necessary. Its physico-chemical properties like molecular weight (475.6 daltons), half-life (1.9-3.7 hours), $\log \mathrm{P}$ value (1.51 at a pH of 7.4), low dose (2mg twice daily) and aqueous solubility $(12 \mathrm{~g} / \mathrm{L}$ at room temperature) make it an ideal drug candidate for transdermal delivery (5). One limitation to transdermal drug delivery is that the drug must be capable of passing through the skin which forms a major barrier to most exogenous substances, including drugs. One approach is to reversibly reduce the barrier function of the skin with the aid of penetration enhancers or accelerants (6). So far there is no published literature report on skin permeation of TT by iontophoresis.

Transdermal iontophoresis is the technique used to enhance the permeation and penetration of drug molecules across the skin using the current density of less than $0.5 \mathrm{~mA} / \mathrm{cm}^{2}$. Chemical enhancers in tandem with iontophoresis increase the transport efficiency and reduce the severity of skin irritation and thereby improve safety of enhancers (7). Gels are considered to be the most suitable delivery vehicle for iontophoresis as they can be easily amalgamated with the iontophoretic delivery system and can also match the contours of the skin (8).

Taguchi method is a combination of mathematical and statistical techniques used in an empirical study which is economical for characterizing a complicated process. It uses fewer experiments to study all levels of input parameters and determines the experimental condition having the least variability as the optimum condition. The variability of a property can be expressed by signal to noise $(\mathrm{S} / \mathrm{N})$ ratio where the experimental condition having the maximum $\mathrm{S} / \mathrm{N}$ ratio is considered as the optimal condition, as the variability of characteristics is in inverse proportion to the $\mathrm{S} / \mathrm{N}$ ratio (9).

In the present study, the synergism of chemical enhancers and iontophoresis was examined by optimizing the levels of four factors (type of enhancer, concentration of enhancer, current density and pulse on/off ratio) on drug skin permeation from gels using Taguchi L16 Orthogonal array method. The efficiency of synergistic effect in overcoming the stratum corneum barrier was studied by in-vivo studies and histological studies.

\section{MATERIALS AND METHODS}

\section{Materials}

TT was obtained as a gift sample from RA Chem. Ltd. (Hyderabad, India). Acrypol-974 was purchased from Corel Pharma Ltd. (Ahmedabad, India). Nerolidol and farnesol was purchased from Alfa Aesar (USA). N-Lauroyl sarcosine was purchased from Hi-media laboratories Ltd (Mumbai, India). Tween-20, ethanol, propylene glycol and triethanolamine were purchased from S. D. Fine-Chem. Ltd. (India).

\section{Preparation of gel}

Gel dosage forms of TT were prepared using a serial mixture of distilled water, propylene glycol (10\%) and ethanol (60\%) as the vehicle and a gelling agent of Acrypol 974 at a concentration of $2 \%(\mathrm{w} / \mathrm{w})$. After complete hydration of Acrypol 974 by the vehicle, drug (0.07\%), penetration enhancer and triethanolamine were added 
and mixed completely, and then the vehicle was added to give a total weight of $100 \mathrm{~g}$. Gel without penetration enhancer was used as a control. Type of penetration enhancer and its concentration added were performed according to the Taguchi L16 orthogonal array experimental design.

\section{Experimental designs}

Taguchi L16 Orthogonal array experimental design was constructed, involving the selection of parameters and the choice of responses. The four factors (independent variables), their corresponding levels and responses (dependent variables) are shown in Table-1. Four factors and their respective levels were selected based on preliminary studies conducted with chemical enhancers. MINITAB 16 software (Minitab Inc., PA, U.S.A) was used for the generation and evaluation of the statistical experimental design.

\section{Ex-vivo permeation studies}

All experiments were conducted according to the protocol approved by the Institutional Animal Ethics Committee (IAEC). A male Wistar rat (180-200g) was sacrificed by excessive ether anesthesia and the hair was removed from the ventral portion using an animal hair clipper (Aesuclap, Germany). After harvesting the full thickness skin, the fat adhering on the dermis side was removed using a scalpel and isopropyl alcohol. Finally, the skin was washed in tap water and stored at $-20^{\circ} \mathrm{C}$ in aluminum foil packing. The skin was used within a week.

Phosphate-buffered saline ( $\mathrm{pH}$ 7.4) was sonicated for $30 \mathrm{~min}$. and placed in the receptor compartment of jacketed Keshary-Chein diffusion cells. The receptor fluids were thermostated at $37 \pm 0.5^{\circ} \mathrm{C}$ and stirred at $600 \mathrm{rpm}$. The thawed skin piece was mounted on the diffusion cell with the stratum corneum side facing the donor compartment and was equilibrated for 1 hour. The gel formulation $(1000 \mu \mathrm{L})$ was applied using a positive pressure pipette to the donor compartment and current (up to $0.5 \mathrm{~mA} / \mathrm{cm}^{2}$ ) was applied either continuously for $6 \mathrm{~h}$ or in periodic fashion through silver-silver chloride electrodes $(2 \mathrm{~cm} \times 0.5 \mathrm{~mm}$ diameter $)$ using a DC regulated power supply unit (K-PAS, Mfg by: M/S. K-PAS Instronic Engineers India PVT LTD., Chennai, India). During periodic iontophoresis, the current was applied using an on/off ratio of 1:3, 1:1, and 3:1 for 6 hours. The anode was placed in the donor compartment and the cathode was placed in the receptor compartment. The samples were periodically withdrawn from the receptor compartment, up to 24 hours, and analyzed by UV-VIS double beam spectrophotometer (Chemito Spectrascan UV2600, India) at $280 \mathrm{~nm}$.

Table 1 - Factors and their corresponding levels implemented for the construction of Taguchi L16 orthogonal array experimental design.

\begin{tabular}{|c|c|c|c|c|c|}
\hline \multicolumn{2}{|c|}{ Factors } & \multicolumn{4}{|c|}{ Levels } \\
\hline & & 1 & 2 & 3 & 4 \\
\hline A & Type of penetration enhancer & Nerolidol & Farnesol & Tween 20 & $\mathrm{~N}$-lauroyl sarcosine \\
\hline B & $\begin{array}{l}\text { Concentration of penetration } \\
\text { enhancer }\end{array}$ & $1 \%$ & $1.5 \%$ & $2.5 \%$ & $5 \%$ \\
\hline C & Current density $\left(\mathrm{mA} / \mathrm{cm}^{2}\right)$ & 0.2 & 0.3 & 0.4 & 0.5 \\
\hline D & Pulse on/off ratio & $\begin{array}{l}0 \text { (continuous } \\
\text { Direct current) }\end{array}$ & $1: 3$ & $1: 1$ & $3: 1$ \\
\hline
\end{tabular}

Response: Amount of TT permeated at 24 hours $\left(Q_{24}, \mu \mathrm{g} / \mathrm{cm}^{2}\right)$,Flux (Jss, $\mu \mathrm{g} / \mathrm{cm}^{2} / \mathrm{hour}$ ) and Enhancement ratio (ER) 


\section{In vivo studies}

The animal study protocol was reviewed and approved by the institutional animal ethics committee. The bioavailability of TT optimized formulation from Taguchi experimental design was compared with oral suspension. Latin square cross over design method was used, where each group consisted of three rabbits. The study was done in two phases; in the first phase group 1 was administered oral suspension and group 2 transdermal gel. In the second phase after 15 days of wash out period vice versa was done: group 2 was administered oral suspension and group 1 transdermal gel.

White New Zealand rabbits weighing 1.5 $\pm 0.50 \mathrm{Kg}$ were selected for the study. The animals were acclimatized to laboratory conditions $\left(25 \pm 1^{\circ} \mathrm{C}\right)$ for 7 days before the experiment. They were allowed free access to food and water, until the night prior to dosing and were fasted for 10 hours. The dorsal surface of each rabbit's trunk was shaved carefully with the help of electrical shaver followed by cleaning with water. The skin was wiped with saline, followed by application of two cylindrical type iontophoretic applicators (rings made of Teflon, $2 \mathrm{~cm}$ i.d. and $3 \mathrm{~cm}$ height) fixed $10 \mathrm{~mm}$ apart using medical grade adhesive. The gel formulation $(1000 \mu \mathrm{L})$ was applied using a positive pressure pipette to one of the Teflon compartments (anode applicator) and a placebo gel was placed in the reference reservoir (cathode applicator). Further, silver-silver chloride electrodes $0.5 \mathrm{~mm}$ in diameter was immersed in each reservoir taking care that electrodes did not touch skin surface and a constant current of $0.5 \mathrm{~mA} / \mathrm{cm}^{2}$ with pulse on/off ratio 3:1 (optimized from Taguchi experimental design) was applied for 2 hours. Blood samples (1-1.5 mL) from a marginal ear vein were collected periodically up to 72 hours and the plasma was separated by centrifugation and further analyzed by liquid chromatography-mass spectrometry (LCMS).

\section{Analytical procedure}

LCMS was used for analising drug content in plasma. Agilent 1200 HPLC system and API4000 triple-quadrupole mass spectrometer equipped with an electrospray source (ESI), operating in positive ion mode was used. Chromatography was performed using X-terra C18 (4.6mm i.d x 50mm) analytical column (Waters, Milford, MA,USA), Acetonitrile $(0.05 \%$ formic acid) as mobile phase at a flow rate of $1.2 \mathrm{~mL} / \mathrm{min}$. Column effluent was introduced into the mass and the run time was $2.0 \mathrm{~min}$. The optimized parameters for mass were curtain gas, gas1 and gas2 (nitrogen) 40, 40 and 60 units respectively; dwell time $200 \mathrm{~ms}$; source temperature $500^{\circ} \mathrm{C}$; ionspray voltage $5500 \mathrm{~V}$. Unit mass resolution was set in both mass-resolving quadrupole Q1 and Q3. Data was analysed by Analyst 1.4.2 software package (MDS Sciex).

\section{Histopathology studies}

Histopathology studies were performed on rat according to protocol approved by institutional animal ethical committee (IAEC). To determine local toxicity of synergistic effect of chemical enhancer and iontophoresis, the optimized formulation with optimized iontophoretic conditions was applied to rat dorsal surface for 6 hours. Skin after treatment was excised and immersed in 50\% neutral formalin solution in saline for fixation until it was subjected to histological processing and examination. The samples were dehydrated with alcohols (70, 80, 95\% and absolute), xylene, then embedded in paraffin blocks, microtomed, deparafinized, rehydrated with graded alcohols and stained with hematoxylin and eosin (H\&E). H\&E slides were observed under dark field light microscope under 200x magnification and compared with control.

\section{Data treatment}

The cumulative amount of TT permeated was plotted against time and the flux was calculated from the straight-line portion of the curve. The enhancement ratio was calculated by dividing the flux value with enhancer to that obtained without enhancer $(10,11)$.

From the plasma concentration-time curve, the pharmacokinetic parameters of $\mathrm{C}_{\max }$ and $\mathrm{T}_{\max }$ were calculated, and the area under the curve (AUC) was calculated using the trapezoidal method. The relative bio-availability was calculated by dividing the AUC of the transdermal gel to the AUC of oral suspension and multiplied by 100 . 
All the experiments were performed in triplicate unless specified, and the data were subjected to one-way ANOVA and t-test at a significance level of $\mathrm{P} \leq 0.05$ using Sigma plot software (Sigma plot 10, USA).

\section{RESULTS}

Using the combinations of the four factors and four levels, formulations were prepared and placed in Keshary-chein diffusion cells, as described previously. The results of the ex-vivo skin permeation studies are shown in Table-2. The data were analyzed to identify the factors that affect statistically significant the responses by $\mathrm{S} / \mathrm{N}$ ratio plots.

Effect of type of penetration enhancer on the skin permeation of TT

Table-2 shows the skin permeation rates $\left(Q_{24}\right)$, flux and enhancement ratio of TT through rat skin from gel formulations. The main effects

Table 2 - Permeation parameters of TT through rat skin from gels used in Taguchi L16 Orthogonal array design.

\begin{tabular}{|c|c|c|c|c|c|c|c|}
\hline \multicolumn{6}{|c|}{ Levels } & \multicolumn{2}{|c|}{ Permeation parameters } \\
\hline $\begin{array}{l}\text { Formulation } \\
\text { code }\end{array}$ & $\begin{array}{c}\text { Type of } \\
\text { penetration } \\
\text { enhancer }\end{array}$ & $\begin{array}{c}\text { Concentration } \\
\text { of penetration } \\
\text { enhancer }(\%, v / v)\end{array}$ & $\begin{array}{l}\text { Current } \\
\text { density } \\
\left(\mathrm{mA} / \mathrm{cm}^{2}\right)\end{array}$ & $\begin{array}{c}\text { Pulse } \\
\text { on/off } \\
\text { ratio }\end{array}$ & $\begin{array}{c}\text { Cum amt, } \\
Q_{24} \pm S . D\left(\mu \mathrm{g} / \mathrm{cm}^{2}\right), \\
n=3\end{array}$ & $\begin{array}{c}\text { Flux, } \mathrm{J}_{\mathrm{ss}} \pm \mathrm{S} . \mathrm{D}(\mu \mathrm{g} / \\
\left.\mathrm{cm}^{2} / \text { hour }\right), \mathrm{n}=3\end{array}$ & $\begin{array}{c}\text { Enhancement } \\
\text { ratio, ER }\end{array}$ \\
\hline CIT1 & 1 & 1 & 1 & 1 & $134.35 \pm 3.92$ & $6.10 \pm 0.21$ & 1.58 \\
\hline CIT2 & 1 & 2 & 2 & 2 & $127.19 \pm 3.08$ & $5.79 \pm 0.09$ & 1.50 \\
\hline CIT3 & 1 & 3 & 3 & 3 & $123.77 \pm 3.68$ & $5.56 \pm 0.21$ & 1.44 \\
\hline CIT4 & 1 & 4 & 4 & 4 & $145.71 \pm 2.00$ & $6.73 \pm 0.03$ & 1.75 \\
\hline CIT5 & 2 & 1 & 2 & 3 & $122.24 \pm 1.42$ & $5.56 \pm 0.09$ & 1.44 \\
\hline CIT6 & 2 & 2 & 1 & 4 & $133.61 \pm 2.82$ & $6.06 \pm 0.10$ & 1.57 \\
\hline CIT7 & 2 & 3 & 4 & 1 & $138.84 \pm 2.90$ & $5.80 \pm 0.10$ & 1.51 \\
\hline CIT8 & 2 & 4 & 3 & 2 & $128.13 \pm 1.99$ & $5.88 \pm 0.16$ & 1.53 \\
\hline CIT9 & 3 & 1 & 3 & 4 & $127.91 \pm 3.19$ & $5.83 \pm 0.11$ & 1.51 \\
\hline CIT10 & 3 & 2 & 4 & 3 & $132.79 \pm 2.57$ & $6.03 \pm 0.19$ & 1.57 \\
\hline CIT11 & 3 & 3 & 1 & 2 & $117.96 \pm 3.46$ & $5.52 \pm 0.09$ & 1.43 \\
\hline CIT12 & 3 & 4 & 2 & 1 & $138.64 \pm 2.20$ & $6.33 \pm 0.10$ & 1.64 \\
\hline CIT13 & 4 & 1 & 4 & 2 & $119.07 \pm 2.65$ & $5.36 \pm 0.10$ & 1.39 \\
\hline CIT14 & 4 & 2 & 3 & 1 & $118.11 \pm 3.87$ & $5.53 \pm 0.27$ & 1.44 \\
\hline CIT15 & 4 & 3 & 2 & 4 & $111.93 \pm 3.39$ & $5.07 \pm 0.19$ & 1.32 \\
\hline CIT16 & 4 & 4 & 1 & 3 & $107.58 \pm 3.22$ & $4.86 \pm 0.18$ & 1.26 \\
\hline
\end{tabular}


plot for SN ratios which was analyzed from $\mathrm{Ta}-$ guchi design, is shown in Figures 1-3A for different response variables. It can be observed that the influence of enhancer type on $Q_{24}$, flux and ER was significant $(p<0.05)$. As observed, gels containing nerolidol resulted in higher permeation followed by farnesol.

\section{Effect of concentration of penetration enhancer} on the skin permeation of TT

From the SN Ratio plots shown in Figures 1-3B the effect of enhancer concentration on $\mathrm{Q}_{24}$, flux and ER was not significant. It can be seen that the maximum permeation rate was obtained with $5 \%$.

Effect of current density on iontophoretic delivery of TT

A linear relationship was observed between current density and permeation rate, shown in Figures 1-3C. Maximum permeation was obtained with maximum current applied at $0.5 \mathrm{~mA} / \mathrm{cm}^{2}$.

Effect of pulse on/off ratio on iontophoretic delivery of TT

The effect of pulse on/off ratio on iontophoretic delivery was investigated since it has a significant effect on iontophoretic delivery $(7,20)$. However, the analysed SN ratio plots showed that the effect of on/off interval was not significant in this study (Figures 1-3D). Maximum permeation was seen with continuous direct current and 3:1 pulse on/off ratio.

Formulation CIT4 (nerolidol 5\%, iontophoretic conditions $\left(0.5 \mathrm{~mA} / \mathrm{cm}^{2}\right.$ and $3: 1 \mathrm{on} /$ off ratio)) was optimized by Taguchi robust design method.

Maximum cumulative amount permeated in $24 \mathrm{hrs}\left(\mathrm{Q}_{24}\right)$ was $145.71 \pm 2.00 \mu \mathrm{g} / \mathrm{cm}^{2}$ by CIT4 followed by CIT7 and CIT12 $\left(138.84 \pm 2.90 \mu \mathrm{g} / \mathrm{cm}^{2}\right.$, $138.64 \pm 2.20 \mu \mathrm{g} / \mathrm{cm}^{2}$ ) respectively. Permeation was least enhanced by CIT16 $(107.58 \pm 3.22 \mu \mathrm{g} /$ $\left.\mathrm{cm}^{2}\right)$ when compared with control $(91.89 \pm 2.30 \mu \mathrm{g} /$ $\mathrm{cm}^{2}$ ). The permeation profile of different formulations is shown in Figure 4 and 5.

Slope of curve $Q_{24}$ Vs time gave flux, and maximum flux was obtained with CIT4 (6.73 $\pm 0.03 \mu \mathrm{g} / \mathrm{cm}^{2} /$ hour $)$ followed by CIT12 (6.33 \pm $0.10 \mu \mathrm{g} / \mathrm{cm}^{2} /$ hour$)$, CIT1 $\left(6.10 \pm 0.21 \mu \mathrm{g} / \mathrm{cm}^{2} /\right.$ hour$)$,
CIT6 (6.06 $\pm 0.10 \mu \mathrm{g} / \mathrm{cm}^{2} /$ hour $)$ and CIT10 (6.03 \pm $0.19 \mu \mathrm{g} / \mathrm{cm}^{2} /$ hour$)$.

CIT4 enhanced permeation by 1.75 fold followed by CIT12 (1.64 fold), CIT1 (1.58 fold), CIT10 and CIT6 (1.57 fold). Lowest enhancement was by CIT16 (1.26 fold).

\section{Pharmacokinetic studies of TT transdermal gel}

Plasma drug concentration vs. time profiles of TT after topical administration of the optimized formulation and oral suspension to three rabbits are shown in Figure-6. Dose of $2 \mathrm{mg}$ TT per $5 \mathrm{~mL}$ suspension was administered orally and for transdermal formulation dose of $0.7 \mathrm{mg}$ TT per $1000 \mathrm{mg}$ of gel was applied. Blood samples were collected only for 24 hours in case of oral formulation as TT half life is 1.9-3.7 hours and its dosing frequency is twice a day where as in case of transdermal as it is sustained release dosage form so samples were collected for 72 hours. Pharmacokinetic parameters calculated from the plasma drug concentration vs. time profiles are listed in Table-3. Maximum concentration of $316.83 \pm 42.40 \mathrm{ng} / \mathrm{mL}$ was obtained with oral suspension when compared with transdermal gel $(176.47 \pm 12.01 \mathrm{ng} / \mathrm{mL})$. Half life $(\mathrm{T} 1 / 2)$ of the formulation was increased from $3.16 \pm 0.18$ hour by oral suspension to $10.88 \pm 0.39$ hours by transdermal gel.

There were significant differences in $\mathrm{C}_{\max }$ and $\mathrm{AUC}_{0-72}$ between the optimized tolterodine gel and the oral suspension. The overall mean value of $\mathrm{AUC}_{0-72}$ by transdermal route was 3.17 times higher than that of oral route, and the difference was found to be statistically significant $(p<0.005)$. The optimized tolterodine gel had a relative bioavailability of $317 \%$ compared to oral suspension.

\section{Histological studies}

The optimized formulation CIT4 and optimized iontophoretic conditions $\left(0.5 \mathrm{~mA} / \mathrm{cm}^{2}\right.$ and 3:1 on/off ratio) were applied to rat dorsal surface and histological studies were conducted.

The H\&E slides of treated skin under 200x magnification shown in Figure-7 showed bleeding, congestion, edema, infiltration of mononuclear cells, degeneration and fatty change in dermis. 
Figure 1 - Main effects plot for SN ratios of (A) type of penetration enhancer, (B) concentration of penetration enhancer, (C) current density and (D) pulse on/off ratio for cumulative amount permeated in 24 hours $\left(Q_{24}\right)$.

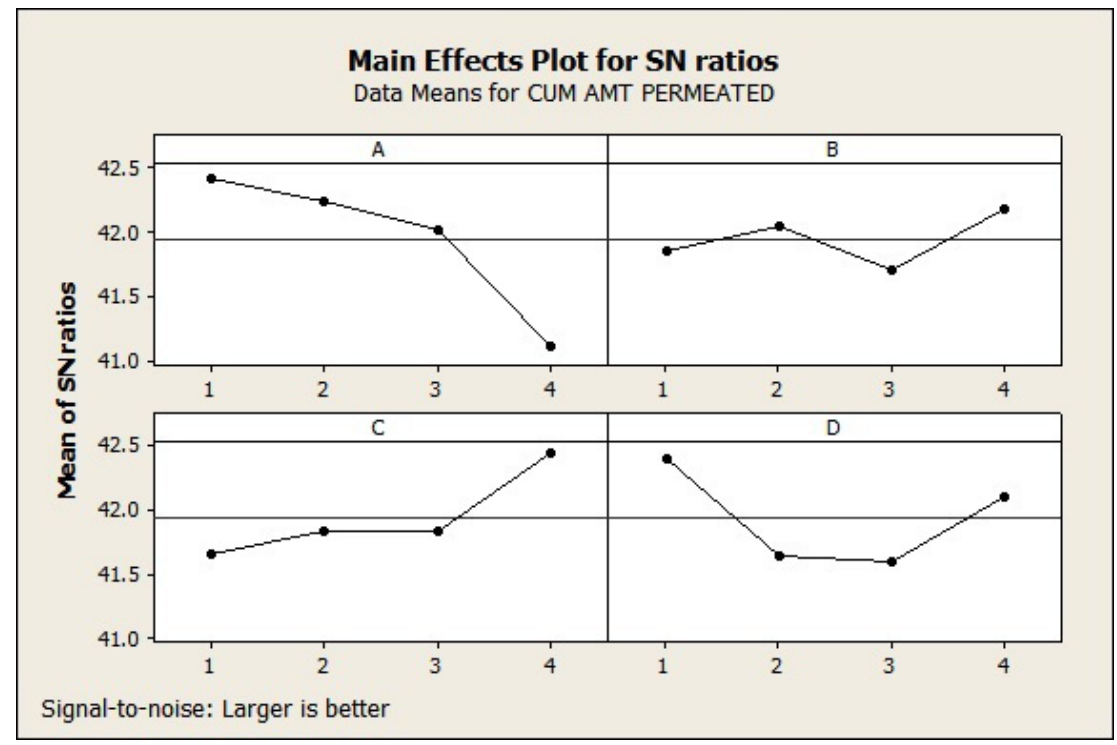

Figure 2 - Main effects plot for SN ratios of (A) type of penetration enhancer, (B) concentration of penetration enhancer, (C) current density and (D) pulse on/off ratio for flux.

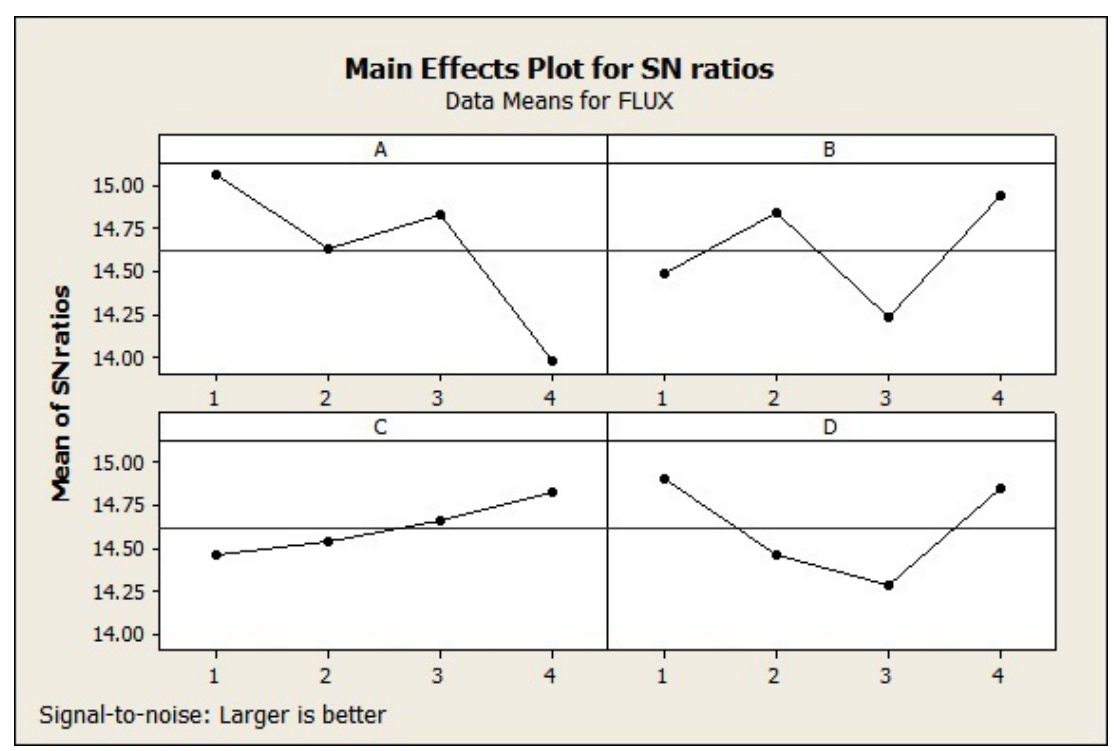


Figure 3 - Main effects plot for SN ratios of (A) type of penetration enhancer, (B) concentration of penetration enhancer, (C) current density and (D) pulse on/off ratio for enhancement ratio.

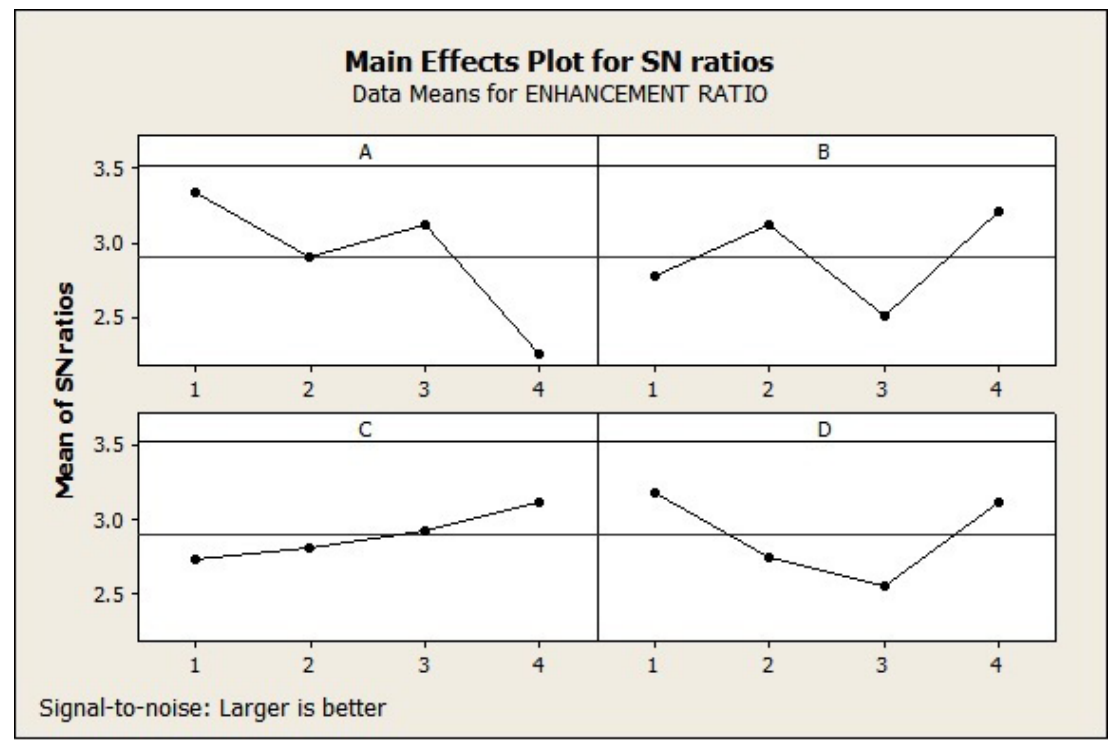

Figure 4 - Permeation profile of formulations CIT1 to CIT8 in comparison with control (BT9).

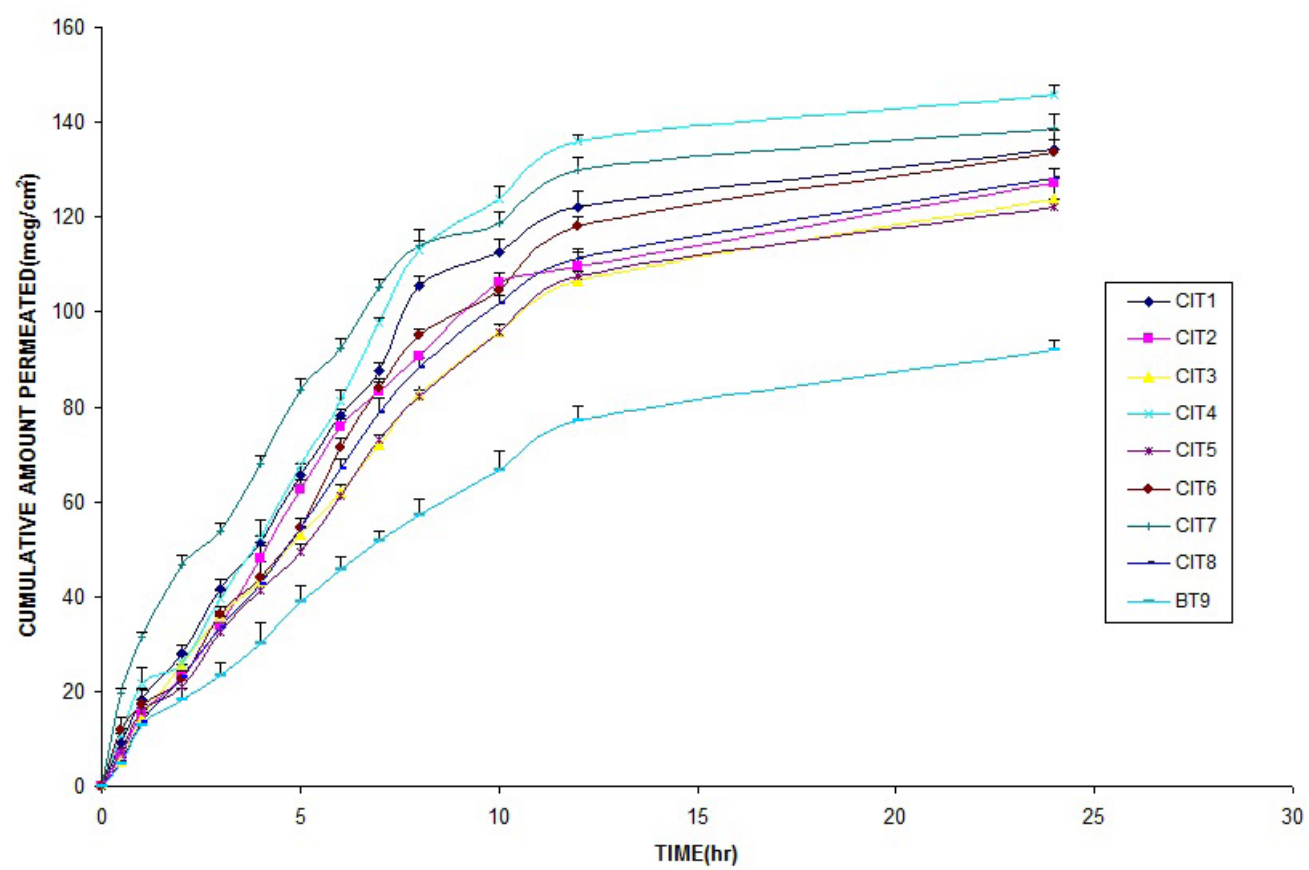


Figure 5 - Permeation profile of formulations CIT9 to CIT16 in comparison with control (BT9).

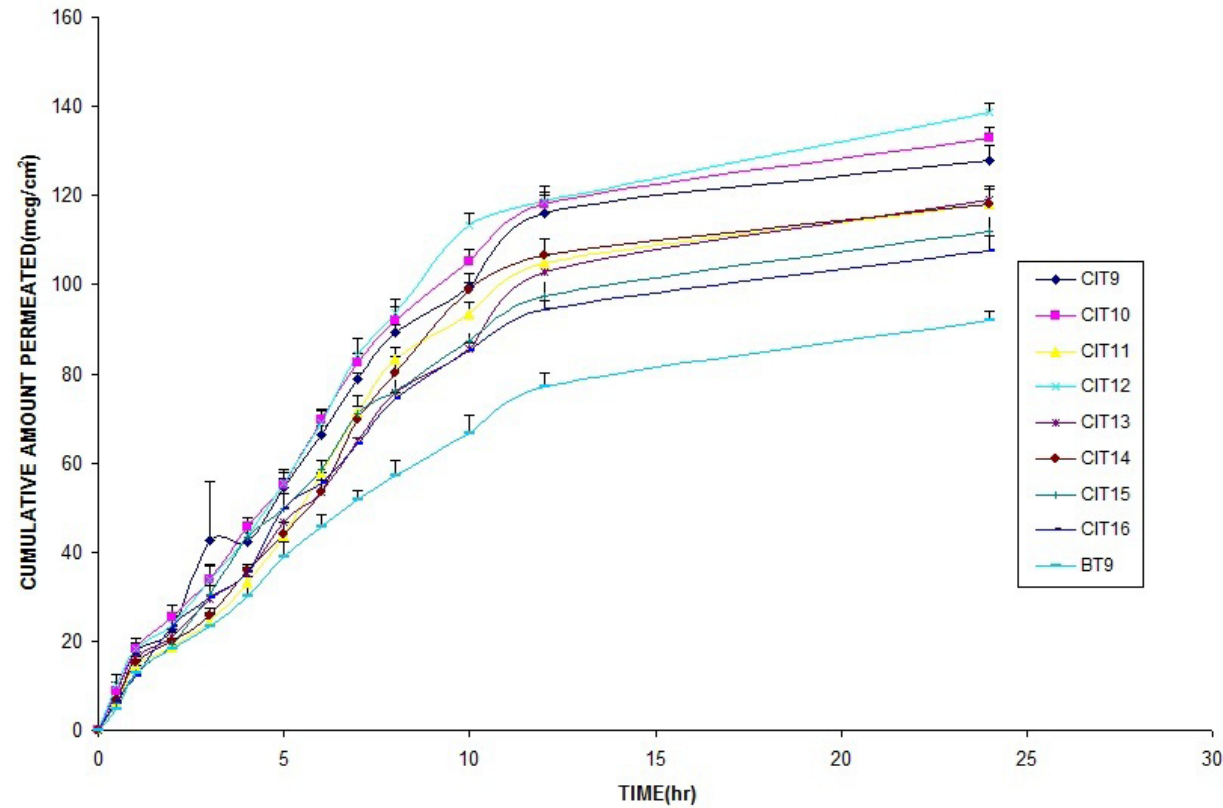

Figure 6 - Plasma concentration-time profiles of tolterodine tartrate in Rabbits after administration of oral suspension and gel with enhancer and iontophoresis, values represented are mean \pm S.D $(n=3)$.

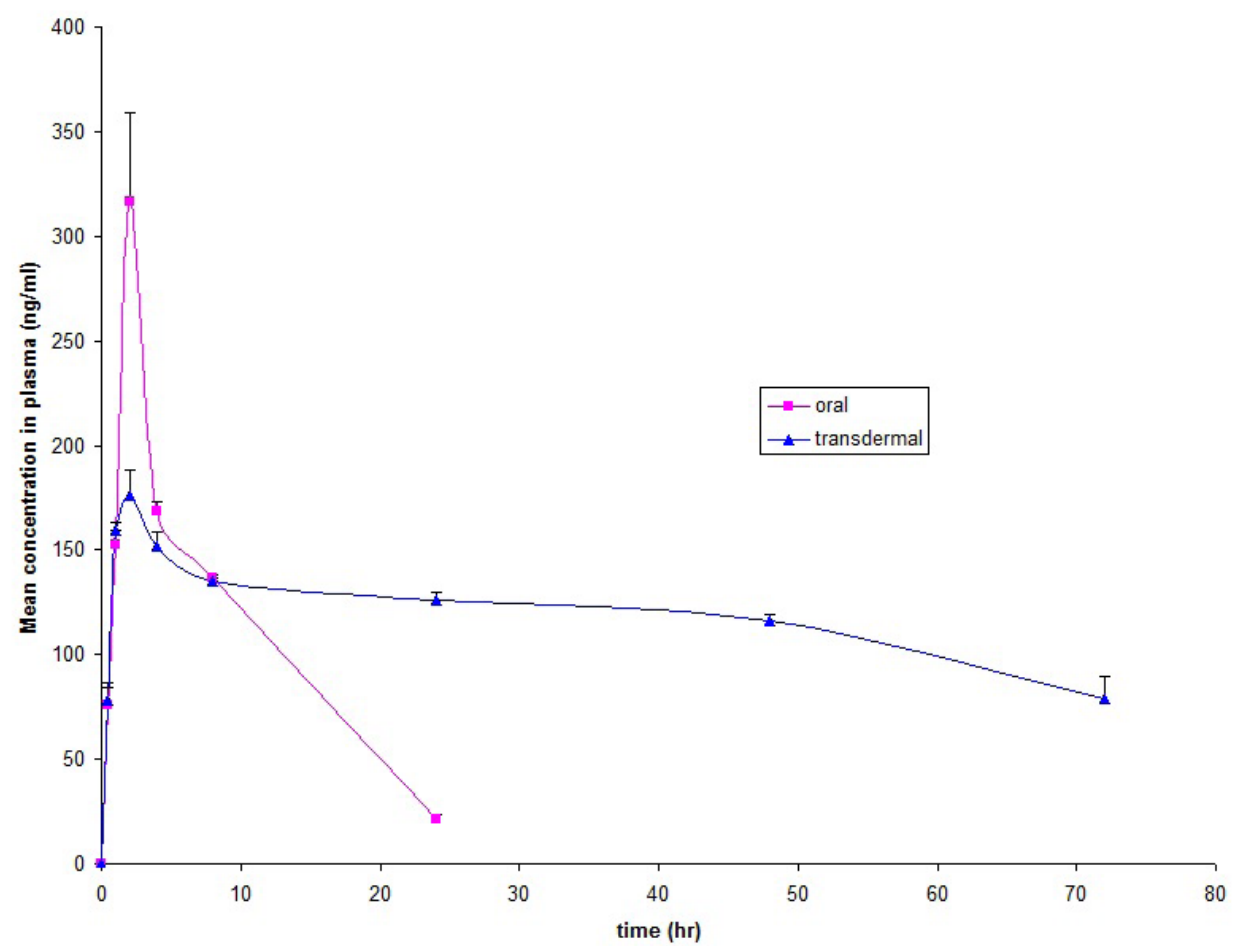


Figure 7 - H\&E sections of skin a) control (untreated) and b) treated with CIT4 and iontophoresis observed under 200x magnification.

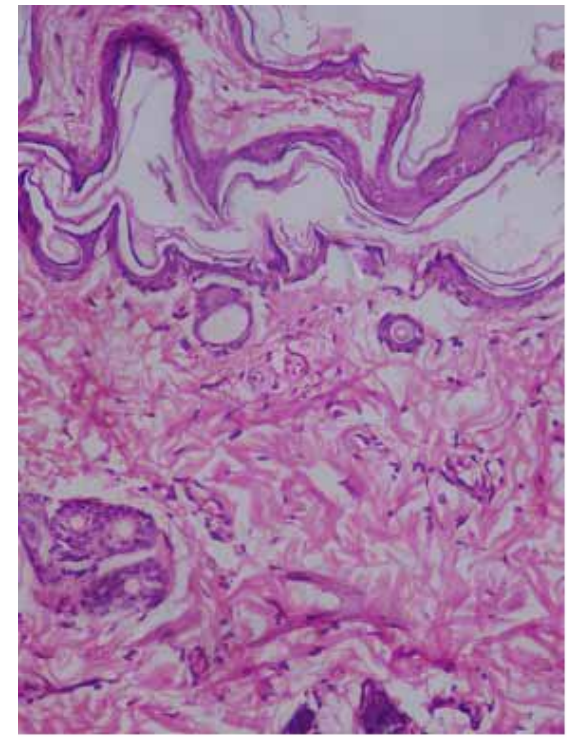

(a)

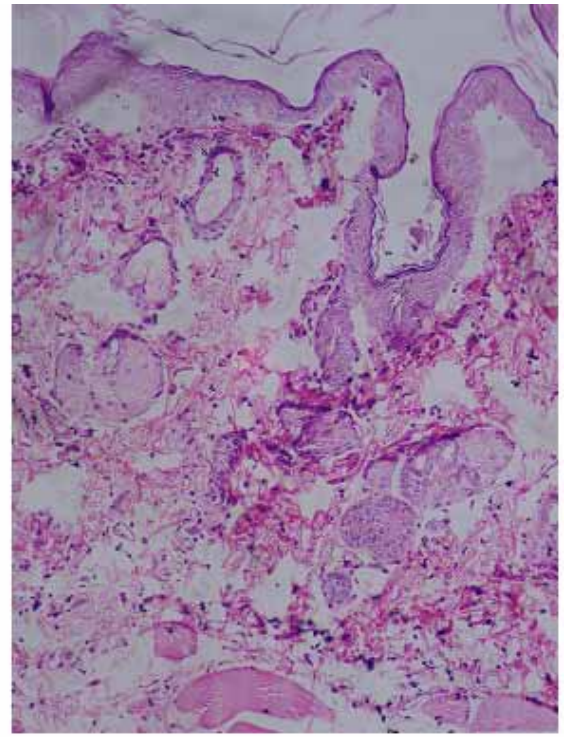

(b)

Table 3 - Mean ( \pm S.D) pharmacokinetic parameters of TT following administration of oral suspension or application of transdermal gel with iontophoresis in Rabbits $(n=3)$. The results were found to be significant $(p<0.005)$.

\begin{tabular}{lcc}
\hline Pharmacokinetic Parameter & Oral suspension & Transdermal gel \\
\hline $\mathrm{C}_{\text {max }}(\mathrm{ng} / \mathrm{mL})$ & $316.83 \pm 42.40$ & $176.47 \pm 12.01$ \\
$\mathrm{~T}_{\text {max }}(\mathrm{h})$ & $2.00 \pm 0.00$ & $2.00 \pm 0.00$ \\
$\mathrm{AUC}_{0-72 \mathrm{~h}}(\mathrm{ng}-\mathrm{h} / \mathrm{mL})$ & $2672.56 \pm 66.00$ & $8477.31 \pm 224.18$ \\
$\mathrm{AUC}_{\text {total }}(\mathrm{ng}-\mathrm{h} / \mathrm{mL})$ & $2770.07 \pm 65.24$ & $10454.13 \pm 332.72$ \\
$\mathrm{~T}_{1 / 2}(\mathrm{H})$ & $3.16 \pm 0.18$ & $10.88 \pm 0.39$ \\
Relative BA $(\%)$ & - & 317 \\
\hline
\end{tabular}

\section{DISCUSSION}

In transdermal drug delivery, overcoming stratum corneum barrier is challenging and the novel method is synergism of chemical and physical enhancer. In the present study, synergistic effect of chemical enhancers and iontophoresis technique on permeation of TT has been determined. In this study, Taguchi robust design was used to optimize the formulation and four factors and four levels were selected from preliminary studies and literature reports by previous researches.

Terpenes (nerolidol and farnesol) were selected based on the reports by researches that 
smaller alcoholic terpenes with a higher degree of unsaturation appeared to be good candidates for enhancing the permeation of hydrophilic drugs (12). And among chemical enhancers, surfactants (tween 20 and N-lauroyl sarcosine) were found to enhance permeation at lower concentrations. It was reported Tween 20 enhanced permeation of 5-fluorouracil by 6-fold (13) and N-lauroyl sarcosine enhanced fluorescein by 47 fold (14).

The maximum current density has been suggested to be $0.5 \mathrm{~mA} / \mathrm{cm}^{2}$ for iontophoresis and the pattern of current application (direct/pulsed) affects the permeation profile (7).

Hence, based on these reports, type of penetration enhancer, concentration of enhancer, current density to be applied and current pattern (pulse on/off ratio) were selected as factors and their effect on response variables were studied by Main effects plots of SN Ratios.

From SN Ratio plots it has been observed that penetration enhancer plays major role and among the penetration enhancers nerolidol showed better enhancement.

For the explanation of the mode of action of terpenes, two mechanisms of permeation enhancement are possible: increased lipid disruption of the stratum corneum by terpenes and the complex formation between the enhancer and drug or structures from the stratum corneum $(15,16)$. Nerolidol and farnesol share similar structural features such as the presence of an alcoholic $\mathrm{OH}$ group that is capable of hydrogen bonding and higher $\log \mathrm{P}$ values which has a linear relationship with enhancement (17), but nerolidol was found to be a better enhancer than farnesol in the enhancement of diclofenac sodium where nerolidol provided 198-fold increase and farnesol 78-fold increase (16). Nerolidol was found to be the most efficient penetration enhancer with respect to four model drugs: nicardipine hydrochloride, hydrocortisone, carbamazepine and tamoxifen $(12,15,18)$.

Tween 20 and N-lauroyl sarcosine are non-ionic and anionic surfactants. In general, the proposed mechanism of surfactants includes fluidization of lipid bilayers, denaturation of keratin, dissolution of skin lipids, disruption of lamellar structure of lipids and swelling of keratinocytes (19). N-lauroyl sarcosine in combination with ethanol synergistically increases skin permeability by increasing the fluidity of stratum corneum lipid structure (14). In the present study, ethanol 60\% was incorporated in base gel. But better enhancement was observed with nerolidol as the combined effects of enhancers and iontophoresis depend on the physico-chemical properties of the penetrant, enhancer and their behavior under the influence of an electric field (7). However, more often synergistic effects have been reported with fatty acids and terpenes.

Concentration of enhancer did not show a linear relationship with enhancement and this fact was in accordance with studies reported with diclofenac sodium (16).

Current density showed a linear relationship with permeation rate, which was also reported by Al-Khalili et al. (20) and Liu et al. (21).

Use of pulsed direct current is recommended over direct current as continuous direct current results in skin polarization, which reduces permeation and to overcome this if pulsed direct current is used it allows depolarization of skin in between as direct current is delivered periodically, thus enhancing permeation (7). But in the present study both direct current and pulse on/off ratio (3:1) showed maximum permeation which could be due to synergistic effect of chemical enhancers.

A computer optimization technique, based on experimental design, has been proven to be a useful approach for selecting pharmaceutical formulations (22-24). Formulation CIT4 (nerolidol $5 \%, 0.5 \mathrm{~mA} / \mathrm{cm}^{2}$ current density and $3: 1$ pulse on/ off ratio) was optimized by Taguchi robust design.

In vivo studies were conducted on rabbit model by Latin square cross over design. Optimized formulation CIT4 (nerolidol 5\%, $0.5 \mathrm{~mA} / \mathrm{cm}^{2}$ current density and 3:1 pulse on/off ratio) effect was compared with standard oral dose as suspension. From the plasma concentrations obtained, there was increase in $\mathrm{T}_{1 / 2}$ by 3.44 times and overall $\mathrm{AUC}_{\text {total }}$ revealed threefold to fourfold increase in bioavailability over oral suspension. These increases in parameters were observed with $0.7 \mathrm{mg}$ dose in transdermal gel when compared with $2 \mathrm{mg}$ dose in oral suspension indicating lower doses are sufficient on delivering drug to systemic circulation. The relative bioavailability of 317\% was obtained 
over oral suspension. These pharmacokinetic parameters indicated efficiency of transdermal gel with initial two cycles of iontophoresis.

The local toxicity and permeation effect of chemical enhancers and iontophoresis were evaluated by histological studies which showed changes in the dermis which can be attributed to the effect of terpenes and ethanol on the lipids. This also reveals severe damage to skin was not caused by iontophoresis. These findings support studies conducted with terpenes and iontophoresis in enhancing insulin (10) and vasopressin permeation (8).

\section{CONCLUSIONS}

Transdermal gels were formulated successfully using Taguchi robust design and these results suggest that type of penetration enhancer affected significantly this process. Nerolidol was optimized as the effective enhancer in combination with iontophoresis. From in vivo studies nerolidol in combination with iontophoresis proved to be an effective method for transdermal delivery of TT. Histological studies showed that the enhancer and iontophoretic conditions applied were safe.

\section{CONFLICT OF INTEREST}

None declared.

\section{REFERENCES}

1. Pfizer: Detrol (TT) tablets prescribing information. New York, NY; 2008.

2. Stewart K, McGhan WF, Offerdahl T, Corey R: Overactive bladder patients and role of the pharmacist. J Am Pharm Assoc (Wash). 2002; 42: 469-76.

3. Guay DR: Tolterodine, a new antimuscarinic drug for treatment of bladder overactivity. Pharmacotherapy. 1999; 19: 267-80.

4. Nilvebrant L, Hallén B, Larsson G: Tolterodine--a new bladder selective muscarinic receptor antagonist: preclinical pharmacological and clinical data. Life Sci. 1997; 60: 1129-36.

5. Vinay Pandit, Aisha Khanum, Shyamala Bhaskaran, Vasiha Banu: Formulation and Evaluation of transdermal films for the treatment of overactive bladder. International journal of Pharm Tech Research. 2009; 1: 799-804.
6. Zhao L, Li Y, Fang L, He Z, Liu X, Wang L, et al.: Transdermal delivery of tolterodine by 0 -acylmenthol: In vitro/in vivo correlation. Int J Pharm. 2009; 374: 73-81.

7. Wang $Y$, Thakur R, Fan Q, Michniak B: Transdermal iontophoresis: combination strategies to improve transdermal iontophoretic drug delivery. Eur J Pharm Biopharm. 2005; 60: 179-91.

8. Nair V, Panchagnula R: Poloxamer gel as vehicle for transdermal iontophoretic delivery of arginine vasopressin: evaluation of in vivo performance in rats. Pharmacol Res. 2003; 47: 555-62.

9. Ki Do Kim, Dae Nam Han, Hee Taik Kim: Optimization of experimental conditions based on the Taguchi robust design for the formation of nano-sized silver particles by chemical reduction method. Chem Eng J. 2004; 104: 55-61.

10. Pillai 0 , Panchagnula R: Transdermal delivery of insulin from poloxamer gel: ex vivo and in vivo skin permeation studies in rat using iontophoresis and chemical enhancers. J Control Release. 2003; 89: 127-40.

11. Jantharaprapap R, Stagni G: Effects of penetration enhancers on in vitro permeability of meloxicam gels. Int J Pharm. 2007; 343: 26-33.

12. Sapra B, Jain S, Tiwary AK: Percutaneous permeation enhancement by terpenes: mechanistic view. AAPS J. 2008; 10: $120-32$.

13. Rigg PC, Barry BW: Shed snake skin and hairless mouse skin as model membranes for human skin during permeation studies. J Invest Dermatol. 1990; 94: 235-40.

14. Kim YC, Park JH, Ludovice PJ, Prausnitz MR: Synergistic enhancement of skin permeability by N-lauroylsarcosine and ethanol. Int J Pharm. 2008; 352: 129-38.

15. El-Kattan AF, Asbill CS, Michniak BB: The effect of terpene enhancer lipophilicity on the percutaneous permeation of hydrocortisone formulated in HPMC gel systems. Int $\mathrm{J}$ Pharm. 2000; 198: 179-89.

16. Nokhodchi A, Sharabiani K, Rashidi MR, Ghafourian T: The effect of terpene concentrations on the skin penetration of diclofenac sodium. Int J Pharm. 2007; 335: 97-105.

17. Kang L, Yap CW, Lim PF, Chen YZ, Ho PC, Chan YW, et al.: Formulation development of transdermal dosage forms: quantitative structure-activity relationship model for predicting activities of terpenes that enhance drug penetration through human skin. J Control Release. 2007; 120: 211-9.

18. Aqil M, Ahad A, Sultana Y, Ali A: Status of terpenes as skin penetration enhancers. Drug Discov Today. 2007; 12: 1061-7.

19. Dwibhashyam VS, Vijaya Ratna J: Chemical Penetration Enhancers-An Update: Indian Drugs. 2010; 47: 5-18.

20. Al-Khalili M, Meidan VM, Michniak BB: Iontophoretic transdermal delivery of buspirone hydrochloride in hairless mouse skin. AAPS PharmSci. 2003; 5: E14. 
21. Liu W, Hu M, Liu W, Xue C, Xu H, Yang X: Investigation of the carbopol gel of solid lipid nanoparticles for the transdermal iontophoretic delivery of triamcinolone acetonide acetate. Int J Pharm. 2008; 364: 135-41.

22. Rhee YS, Chang SY, Park CW, Chi SC, Park ES: Optimization of ibuprofen gel formulations using experimental design technique for enhanced transdermal penetration. Int J Pharm. 2008; 364: 14-20.
23. Agyralides GG, Dallas PP, Rekkas DM: Development and in vitro evaluation of furosemide transdermal formulations using experimental design techniques. Int J Pharm. 2004; 281: $35-43$.

24. Ahad A, Aqil M, Kohli K, Sultana Y, Mujeeb M, Ali A: Formulation and optimization of nanotransfersomes using experimental design technique for accentuated transdermal delivery of valsartan. Nanomedicine. 2012; 8: 237-49.

Correspondence address:

Dr. P. K. Lakshmi

G. Pulla Reddy College of Pharmacy Osmania University, Hyderabad, 500-028, India E-mail: drlakshmisuresh@gmail.com 


\section{EDITORIAL COMMENT}

Muscarinic receptors antagonists, associated or not to behavior therapy are the mainstay first line treatment for overactive bladder. Along the past decade we have observed the launch and approval of different oral antimuscarinics, all of them with some specific receptor target in the detrusor and urotelium. Besides this molecular specificity a large amount of patients taking this medication abandons it in up to six months after beginning the treatment (1). Two major conditions are related to this high discontinuation rate of the oral therapy: the discrepancy between subjective and objective good outcomes, and the frequent adverse events. Most of these undesirable symptoms after antimuscarinic intake are consequences of its hepatic metabolism. The challenge of developing a new way to administer the medication, avoiding the first-pass metabolism has been tried for many years. It started in the 80's with the intravesical instillation, followed by rectal, vaginal and more recently transdermal delivery route (2).

The aim of the present study comes toward the above described scenario as it associates Iontophoresis and enhancers substances to improve the Tolterodine Tartrate absorption through the skin. Although an equivocal defini-

\section{REFERENCES}

1. Shaya FT, Blume S, Gu A, Zyczynski T, Jumadilova Z: Persistence with overactive bladder pharmacotherapy in a Medicaid population. Am J Manag Care. 2005; 11 (4 Suppl): S121-9.

2. Dmochowski RR, Starkman JS, Davila GW: Transdermal drug delivery treatment for overactive bladder. Int Braz J Urol. 2006; 32: 513-20. tion for $\mathrm{OAB}$ had been adopted by the authors (detrusor involuntary contractions are not observed in all patients that complain of OAB syndrome) the perspective of a new option of administration deserves special attention. Other studies in the literature presented good clinical results with transdermal use of antimuscarinics (3). Something new this experimental study may add to the other various publications regarding this issue is the positive synergic effect of Iontophoresis and the enhancer Nerolidol. The study opens the perspective to obtain an adequate and persistent level of Tolterodine in the plasma (demonstrated in the present study) and probably in the bladder wall (not tested in this study).

Some methodological limitations observed in this study as the absence of comparison among the iontophoresis plus gel only and the enhancer (Nerolidol) added; use of two different animals for ex-vivo and in-vivo studies (number of studied animals not cited in the text), and the lack of a dosage of the level of the antimuscarinic in the urine, must be considered when analyzing the presented results.

In face of this, other complementary studies in the same direction are welcome to better demonstrate the usefulness of Iontophoresis plus enhancers for the transdermal use of antimuscarinics.
3. Gomelsky A, Dmochowski RR: Oxybutynin gel for the treatment of overactive bladder. Expert Opin Pharmacother. 2012; 13: 1337-43.

\section{Dr. José Carlos Truzzi} UNIFFESP

R. Sena Madureira, 1500 Vila Mariana, Sao Paulo, 04021-001, Brazil E-mail:jctruzzi@hotmail.com 